EPJ Web of Conferences 41, 01017 (2013)

DOI: $10.1051 /$ epjconf/20134101017

(C) Owned by the authors, published by EDP Sciences, 2013

\title{
Toward a "Perfect-Wave" HHG Driving With a Multicolor OPA
}

T. Balčiūnas ${ }^{1}$, S. Haessler ${ }^{1}$, G.Y. Fan ${ }^{1}$, G. Andriukaitis ${ }^{1}$, A. Pugžlys ${ }^{1}$, A. Baltuška ${ }^{1}$, A. Zaïr ${ }^{2}$, R. Squibb ${ }^{2}$, L. Chipperfield ${ }^{2}$, L. Frasinski ${ }^{2}$, J. W. G. Tisch ${ }^{2}$, J. Marangos $^{2}$

${ }^{1}$ Photonics Institute, Gußhausstraße 27-387, A-1040, Vienna, Austria

${ }^{2}$ Imperial College London, United Kingdom

\begin{abstract}
We realize a multicolor, multi-cycle combination of commonly CEP-locked three waves from a single femtosecond OPA and report HHG driving with individual and combined colors. Employing a novel multicolor multi-cycle incommensuratefrequency waveform synthesizer we demonstrate a previously theoretically predicted capability to enhance the cut-off position of the HHG spectrum and upscale the XUV flux as compared to single-color driving.
\end{abstract}

\section{Introduction}

The cut-off photon energy in high-order harmonic generation (HHG) is famously governed by the $3.17 U_{\mathrm{p}}+I_{\mathrm{p}}$ formula [1], where the factor 3.17 corresponds to a returning electron trajectory for a sinusoidal laser driver field. Recently, the Imperial College London group put forward a theoretical proposal [2] suggesting the use of a multicolor multi-cycle driver waveform that can satisfy the condition of a returning trajectory and, simultaneously, accelerate the tunneled-out electron to a higher kinetic energy compared to the case of a single-color sinusoidal driver field. The initial suggestion for this optimized re-colliding electron steering, dubbed "the perfect wave" [2], assumed a combination of UV optical harmonics of a $800-\mathrm{nm}$ laser and included a near-IR sub-harmonic $(1600 \mathrm{~nm})$ to demonstrate the feasibility of the cut-off enhancement numerically. In this contribution, we present a very efficient and technically straightforward approach to adding Fourier components at octave frequency spacing and demonstrate preliminary results of HHG driving with individual colors of our multi-color synthesizer.

a)

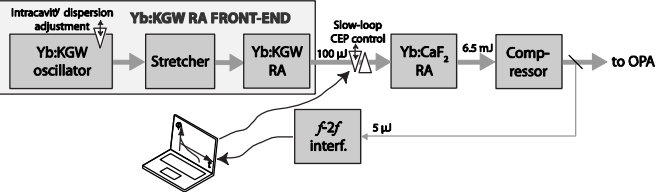

b)

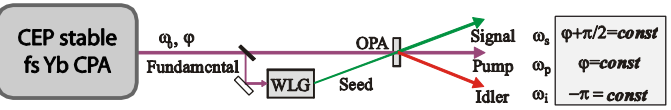

Fig. 1. Concept of a three-wave CEP-stable synthesizer for HHG driving. a) a multi-mJ diode-pumped femtosecond 1- $\mu \mathrm{m}$ CEP-stabilized pump laser; b) collinear mid-IR CEP-stable OPA;

Our concept, depicted in Fig.1, is based on the use of a single collinear optical parametric amplifier (OPA) to serve as a source of three colors that are mutually locked to a single carrierenvelope phase (CEP). The CEP stabilization requirement in waveform synthesis automatically arises for combinations of two and more different-color pulses whenever the mechanism of color multiplication is other than direct optical harmonic generation of the fundamental frequency pulse 
[2]. The main advantages of the OPA-based scheme for perfect-wave synthesis over the color addition by direct consecutive harmonic generation [3] are outlined schematically in Fig.1c and explained as follows:

Firstly, the OPA down-converts the pump frequency to the signal and idler waves rather than to up-convert it as in the harmonic generation approach. Downshifting the base frequency into mid-IR (idler wave) allows us provide room for more non-UV Fourier components and thus avoid problems atmospheric absorption of driver pulses and enhanced multiphoton UV ionization of the HHG target. This is clearly understood from comparing the $4^{\text {th }}$ harmonic wavelength of a Ti:sapphire laser (200 $\mathrm{nm})$ to the $4^{\text {th }}$ harmonic of the idler pulse $(750 \mathrm{~nm})$.

Secondly, the production efficiency of successive optical harmonics via sum-frequency generation rapidly plunges with the harmonic order. In an OPA, similar intensities at the frequencies of the three mixing waves, pump $\left(\omega_{3}\right)$, signal $\left(\omega_{1}\right)$, and idler $\left(\omega_{2}\right)$ are easily ensured and two additional Fourier components are easily added by frequency-doubling $\omega_{3}$ and $\omega_{1}$.

Thirdly, CEP stability of the three OPA waves is ensured by the use of a CEP-stable pump laser and a white-light seed. In such a way, the CEP stability of the pump wave is inherited by the signal through the process of supercontinuum generation and the CEP stability of the idler results from the difference-frequency generation process [4]. Thus the scheme presented in Fig.1b becomes a wastefree multi-octave optical synthesizer seamlessly tunable across commensurate as well as incommensurate frequency combinations.

Previously, we have introduced the concept of a three-wave OPA synthesizer and proved its ability to drive above-threshold ionization and plasma $\mathrm{THz}$ generation experiments [3]. However, the experiments on multicolor HHG driving have remained out of reach because of the low pump pulse intensity from a diode-pumped $\mathrm{Yb}: \mathrm{KGW}$ amplifier. Scaling of the pulse intensity presents a bottleneck in the KGW technology and is determined by parasitic nonlinearities. This contribution describes a radical improvement in the pump laser technology which has allowed us to upgrade the parametric synthesizer to the required intensity level for multicolor HHG driving.

\section{Experimental setup}

The general scheme of the laser amplifier system is depicted in Fig. 1 (a). An Yb:KGW CPA laser system (Light Conversion Ltd.) is used as a front-end for a high energy $\mathrm{Yb}: \mathrm{CaF}_{2} \mathrm{RA}$. The front-end CPA amplifier delivers pulses stretched to $200 \mathrm{ps}$ at up to $6 \mathrm{~W}$ of average power at $10-200 \mathrm{kHz}$ repetition rate and up to $1 \mathrm{~mJ}$ pulses at $1 \mathrm{kHz}$, however, for seeding only $100 \mu \mathrm{J}$ were actually used. For the second stage a cryogenically cooled $\mathrm{Yb}: \mathrm{CaF}_{2} \mathrm{RA}$ is used to amplify the pulses up to $6 \mathrm{~mJ}$. Using a PLL, the carrier-envelope-offset phase of the amplifier chain is actively stabilized. The resulting r.m.s. phase jitter is from the first stage amplifier is $0.45 \mathrm{rad}$ and the typical jitter of the CEP after the second stage RA is approximately $\sigma=0.95 \mathrm{rad}$. Optimization of the second RA cavity is underway in order to improve the stability and reduce the CEP jitter.

\section{High-order harmonic generation results}

The results of the HHG driven by three incommensurate frequency fields are summarized Fig 2. Our preliminary data show the utility of the developed system as a means of significantly increasing the cut-off frequency and overall efficiency of HHG driven by multi cycle pulses. Firstly, HHG was tested using 180 -fs $1.03-\mu \mathrm{m}$ pulses directly from the $\mathrm{Yb}$ laser. Secondly, as a demonstration that the peak power of the system is sufficient for energy demanding applications such as HHG, we measure HHG in argon driven by two waves of our synthesizer individually: the signal wave at $1.545-\mu \mathrm{m}$ of a KTA OPA and the fundamental wave at $1.03 \mu \mathrm{m}$, as shown in Fig. 2. Due to a three times larger periodicity as compared to $1-\mu \mathrm{m}$ wave and asymmetric half-cycles, the density of the harmonic peaks increases six times. Since the amplitude of the individual peaks drops roughly only by a factor of 2 , the total flux integrated over the spectrum of the denser-spaced harmonics increases dramatically. Moreover, the spectrum generated using the two-color driver extends beyond the transmission edge of aluminum filter, demonstrating that the cut-off can be pushed above the 
saturation limit of the $1-\mu \mathrm{m} 180$ fs pulse. When the third color pulse is added, the HHG spectrum becomes highly CEP-dependent and exhibits phase-dependent cut-off modulation. This CEP dependence signifies that sub-cycle shaping is a potent tool for controlling the dynamics of the electron in a strong laser field. Superposition of these different infrared colors with comparable amplitudes provides a route towards a source of high XUV photon energy and flux.
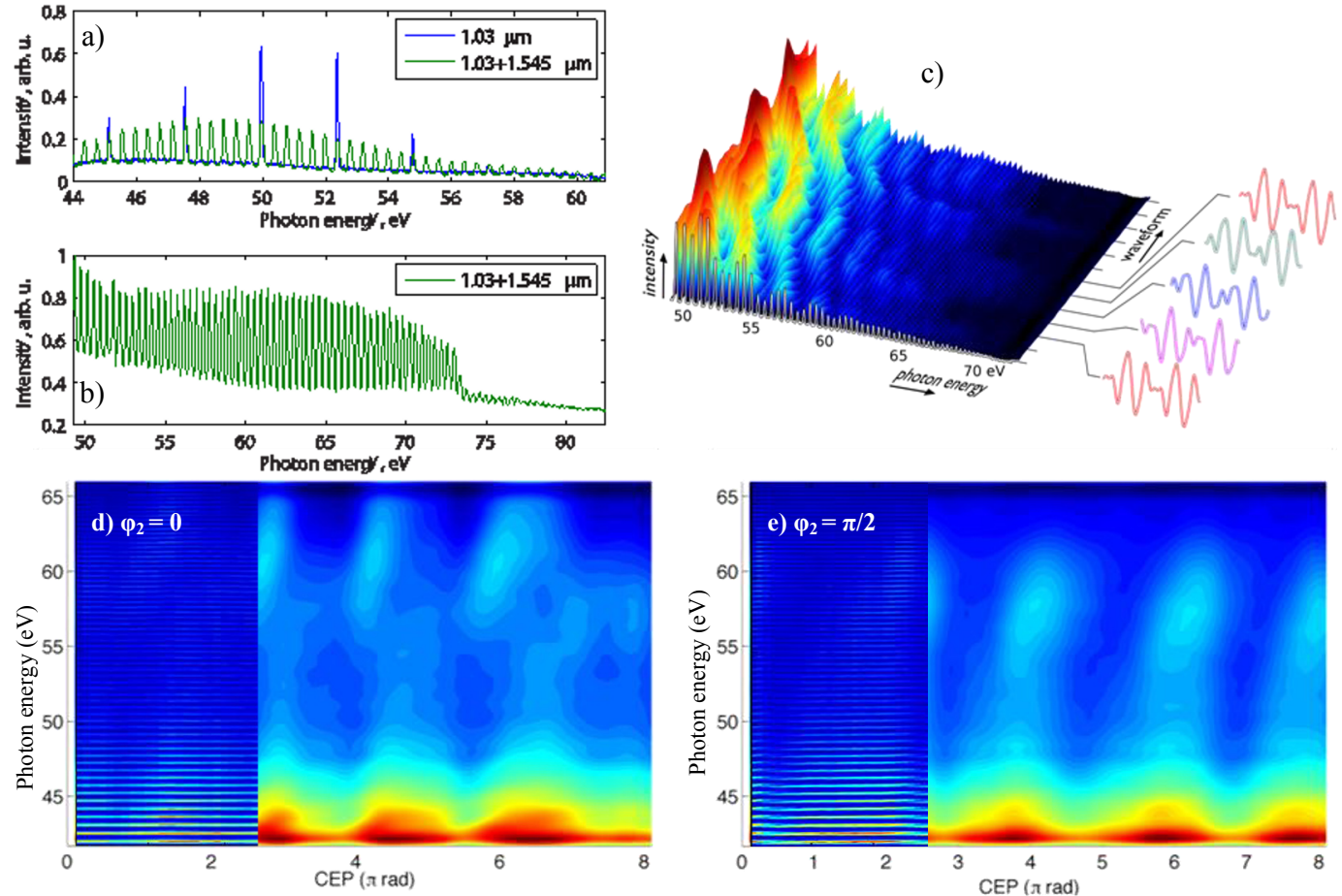

Fig. 2. High order harmonic spectra generated with phase-locked multiple color pulses. a) comparison of HHG spectrum generated in Argon using single color at $1.03 \mu \mathrm{m}$ and two color field composed of $1.03 \mu \mathrm{m}(\omega)$ and $1.545 \mu \mathrm{m}(\omega / 1.5)$ waves. b) Cutt-off region of the two-color spectrum that extends beyond the Al filter transmission edge at $72.5 \mathrm{eV}$. c) a series of measured XUV high-order harmonic spectra generated using three colors, which depend upon the shape of the optical cycle of the generating intense infrared synthesized waveforms. The waveform is controlled by scanning the CEP of the driving laser pulse. d) and e) CEP dependences of low-pass Fourier filtered HHG spectra for different values of $\boldsymbol{\varphi}_{2}$. Insets show raw HHG spectra. Note that low-pass Fourier filtering removes harmonic peaks corresponding to once-per-cycle emission helping to reveal faster, sub-cycle dynamics.

\section{Conclusions}

In conclusion, we present a high-power multi-color source in which the CEP of each color is locked and demonstrate high order harmonic generation with three colors of the system. The control of the maximum recollision energy (cut-off position) and efficiency of the HHG via shaped waveform is demonstrated by recording XUV spectra generated in Argon for different CEP and relative phases.

\section{References}

1. P. B. Corkum, Phys. Rev. Lett. 73, 1994 (1993).

2. L. E. Chipperfield et al., Phys. Rev. Lett. 102, 063003 (2009).

3. T. Balčiūnas, et al., in Int. Conference on Ultrafast Phenomena, p. PDP4

4. A. Baltuška et al., Phys. Rev. Lett. 88, 133901 (2002).

5. A. Pugžlys et al., Appl. Phys. B 97, 339-350 (2009).

6. T. Balčiūnas, et al., Opt. Lett. 36, 3242-3244 (2011). 